\title{
EDITORIAL
}

\section{CURRENT COVID-19 SURGES IN BANGLADESH 2021}

\author{
MA FAIZ
}

Almost one year after the detection of first case of SARS COV-2, Bangladesh witnessed consecutive four days' of more than 7000 cases per day with more than 60 deaths as of $7^{\text {th }}$ April 2021 and more than 100 deaths per day as of $19^{\text {th }}$ April, 2021. Diagnostic capacity by RT-PCR was increased, although rapid antigen detection could be widely used and scaled out for quick diagnosis. Based on the WHO COVID-19 Strategy National Preparedness and Response Plan for COVID19 was developed in early 2020 to prevent and control the pandemic. 'National' plan was not found to be widely available and consulted for implementation by different organs of the government as a part of 'Wholeof Government' approach of containment. The potential reasons behind recent COVID surges in Bangladesh could be:

- Multiple factors and complicated

- New variants of concern (UK, South Africa, Brazil)

- Local/Bangladesh mutations could be responsible too. Lack of adequate genome sequencing research in Bangladesh

- $\quad$ NOT optimal following the public health measures

- COVID denial

- COVID fatigue

- Infodemic - misinformation, rumours.

\section{What can be done?}

Following the strict public health measures -wearing mask appropriately, hand washing, physical distancing, get tested, and get vaccinated. In the initial phase of the pandemic despite denial, there was lots of enthusiasm and community awareness soon died down to adhere the fundamental principles of 'Public Health'. Continued stress on the importance of physical distancing, wearing mask and hand washing was mostly missing and hardly any role model was enthusiastically promoted. Despite a good rural community health care system we are yet to utilize them in taking the opportunity of tackling the pandemic through creating a 'community movement' of health promotion and disease prevention centering COVID-19. Bangladesh developed an excellent strategy for Social and Behaviour Change Communication (SBCC) for creating community awareness on different priority public health problems under different operation plan using the principles of Bangladesh SBCC strategy- 'coordination, capacity development and community engagement' through an implementation plan using Socio-ecological Model (Fig.-1). ${ }^{2}$ This is the right time to test the strategy through developing and implementing COVID-19 SBCC strategy which will strengthen the community system taking example of four already existing community groups for $\sim 6000$ population in the catchment area of a community clinic (CC)- community group and community support groups. ${ }^{3}$

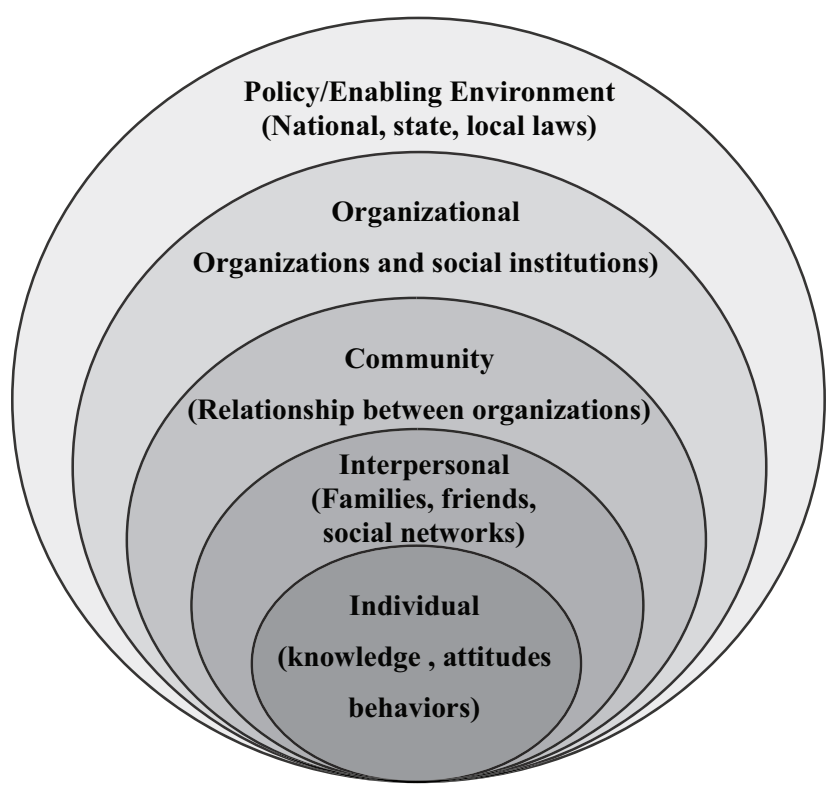

Source: Comprehensive Social and Behavior Change Communication Strategy, MOH\&FW 2016

Fig.-1: Socio-Ecological Model

Initially COVID-19 started in cities having inequal access of health care services to different segment of population. So it is high time to find a viable urban

Professor of Medicine (Retired), Former Director General of Health Services \& Dev Care Foundation, Dhaka, Bangladesh, E-mail: drmafaiz@gmail.com 
primary health care (equivalent to rural $\mathrm{CC}$ based community based health care) in an environment of a non functional urban primary care in Bangladesh when an urban health strategy and action plan recently developed is yet to be implemented. ${ }^{4}$

Vaccine deployment plan was developed based on WHO document which emphasised the vaccination as continuation of the broader COVID strategy which was not discussed in broader community not also visualized as an additional layer of protection not the only way of protection. ${ }^{5,6}$

Communication on different facets of public health measures was there, the reasons of not following them to have an impact is a concern. Is it that we could not propagate the message in the way the community wanted or could understood. Could more anthropological and social involvement could be utilized.The widespread 'rurmours' could be listened to for efficient handling of how to deal with misinformation. Time is not running out to tackle systematically the misinformation prevailing or continue to prevail.

We started the activities for potential pandemic preparedness in 2019 which covers measures at point of entry, few documents were quickly developed. It was unfortunate that 'science' could not be upheld while adherence/compliance to the measures were said to be violated specially mistakes recurred in ensuring mandatory recommended quarantine of the returnees, not enough resources could be mobilised to this end resulting in paying heavy price by the Community.

Lockdown- debatable how effective in adense populated, poor country like Bangladesh.Documents prepared without enough attempts to implement in a concerted manner. Unplanned lockdowns (as we have done so far) rather contributed in increase transmission all over the country via population movement and mismanagement. While writing this note border closure with India to contain the spread is appreciated.

Herd immunity via vaccination - vaccine coverage, vaccine hesitancy are concerns in Bangladesh. 'Herd immunity' through vaccination is the ultimate aim. In order to understand the progression of population immunity antibody level to be measured periodically.

Contact tracing - appears to be missing in Bangladesh. Whatever tests we did was not linked with patients in most of the occasion. In some places we enthusiastically started but soon loosened the contact tracing for quarantine and even isolation of the cases. No dedicated group was responsible for monitored contact tracing and isolation of the cases. The knowhow of 'contact tracing' learned for TB, Nipah, Influenza, Japanese encephalitis was not attempted to use in the
Pandemic. When resurgence was suspected the contact tracing was loosened and gave up rather.

Strengthening testing and tracing. Currently no system in place to identify asymptomatic carriers. We have no clear idea about the percentage $\%$ of population affected in rural, urban and slum areas.

Research capacity: tracking variants of concern (UKB117; SA- B1.351; Brazil- P1) and genome sequencing. Detection of variants either of UK, Brazil, or South African or any new specific to Bangladesh will be possible by genome sequencing. ${ }^{7}$ Capacity development for sequencing and surveillance is urgent need to provide evidence based information and taking decision including currently used vaccine utility. Whatever data is available should be periodically make publicly available with decision.

Evidence based resource allocation. Our resources are very limited - hospital beds, ICUs, trained professionals. Management of highly contagious infections deserve specialized trained infectious disease (ID) medical professionals which was not available in Bangladesh. In order to save the non COVID cases dedicated ID professionals should be deployed in major hospitals across the country.

Infection prevention and control (IPC) a basic element of controlling respiratory infections deserves due attention for controlling the current pandemic, tackling antimicrobial resistance and so forth. Involvement of all health care professionals giving due recognition of services of all health care staff can not be ignored. Outsourcing of basic staff (for example, cleaners, and MLSS, a task specific specialization should not be ignored) essential for IPC including in-house waste management needs to be seriously revisited so also top priority on community waste management deserve due coordinated attention and funding.

\section{References:}

1. DGHS (2020). National Preparedness and Response Plan for COVID-19, Bangladesh. Version 5.

2. MOHFW (2016). Comprehensive Social and Behavior Change Communication Strategy (SBCC).

3. MOHFW (2019). Bangladesh National Strategy for Community Health Workers 2015.

4. MOHFW (2014). National Urban Health Strategy, Bangladesh.

5. WHO (2020). Guidance on developing a national deployment and vaccination plan for COVID-19 vaccines. Interim Guidance.

6. MOHFW (2020). National Deployment and Vaccination Plan for COVID-19 Vaccines in Bangladesh

7. Rapid Risk Assessment: SARS-CoV-2 - increased circulation of variants of concern and vaccine rollout in the EU/EEA, $14^{\text {th }}$ update. 15 February 2021. 